Case Report

\title{
Aseptic Splenic Abscess as Precursory Extraintestinal Manifestation of Inflammatory Bowel Disease
}

\author{
Joel Brooks ${ }^{1}$ and Gisoo Ghaffari ${ }^{2}$ \\ ${ }^{1}$ Heart of Lancaster Regional Medical Center, Department of Medical Education, 1500 Highlands Drive, Lititz, PA 17543, USA \\ ${ }^{2}$ Penn State Hershey Medical Center, Department of Medicine, 500 University Drive, Hershey, PA 17033, USA \\ Correspondence should be addressed to Joel Brooks; joelpbrooks@gmail.com
}

Received 14 July 2014; Revised 26 August 2014; Accepted 26 August 2014; Published 7 September 2014

Academic Editor: Gianfranco D. Alpini

Copyright (C) 2014 J. Brooks and G. Ghaffari. This is an open access article distributed under the Creative Commons Attribution License, which permits unrestricted use, distribution, and reproduction in any medium, provided the original work is properly cited.

Splenic abscesses are most often secondary to aerobic bacterial infections due to Streptococcus, Staphylococcus, and Enterococcus species of organisms. Sterile splenic abscesses rarely occur and diagnosis and treatment of those are challenging. We report a case of a previously healthy young female presenting with aseptic splenic abscesses as the initial manifestation of Crohn's disease along with a review of the literature on aseptic splenic abscess as an extraintestinal manifestation of Crohn's disease.

\section{Introduction}

Splenic abscesses are rare occurrences and are most commonly caused by bacterial infections with an incidence between 0.14 and $0.7 \%[1,2]$. There are fewer than 800 cases reported in the literature regarding splenic abscess. Aseptic splenic abscesses are much less common, with the majority of cases reported from Europe [3-5]. The majority of splenic abscesses are infectious; blood and aspirated fluid cultures often reveal the causative pathogen [6-8]. Aerobic bacteria, Streptococcus, Staphylococcus, Enterococcus, in addition to Escherichia coli (anaerobic) species, have been the causative organisms in most reports [9-12].

This report describes a young female with recurrent aseptic pustular skin lesions as well as sterile splenic abscesses refractory to antibiotic treatments. The small and large bowel biopsies as well as serology suggested Crohn's disease. In addition, the clinical, laboratory, and radiographic responses to prednisone and azathioprine offered additional support for the diagnosis of an inflammatory bowel disease (IBD).

\section{Case Presentation}

A 19-year-old Caucasian female residing in Pennsylvania presented to her primary care provider with pustular lesions on her right ankle. It was suspected that the patient developed cellulitis following a minor blunt trauma to the area. The skin lesions progressed despite outpatient treatment with clindamycin, requiring hospitalization. Significant laboratory findings included the following: elevated white blood cells (WBC; 15.1 cells/microL), erythrocyte sedimentation rate (ESR; 80), and C-reactive protein (CRP; 7.6). Wound and blood cultures along with a right ankle joint aspiration were negative, as well as magnetic resonance imaging (MRI) of the bone and indium-labeled WBC scan for osteomyelitis. The antibiotic was changed to intravenous vancomycin followed by oral clindamycin plus trimethoprim-sulfamethoxazole for outpatient treatment. Two weeks after completion of this therapy, the patient presented with similar lesions on the dorsum of the left foot. Bone MRI showed microabscesses that were incised yielding E. coli from a single culture. She was subsequently treated with 5 weeks of ciprofloxacin and vancomycin for E. coli/Methicillin Resistant Staphylococcus aureus (MRSA) infection.

Three weeks after completing antibiotic treatment, she presented with abdominal pain that was attributed to a urinary tract infection. When the pain did not resolve with empiric antibiotic treatment, an abdominal CT scan was performed which revealed multiple low-density splenic lesions, the largest of which measured $4 \times 2.9 \mathrm{~cm}$ (Figure 1). The antibiotic was changed to intravenous ceftriaxone, followed by daptomycin, ciprofloxacin, and metronidazole. A repeat 


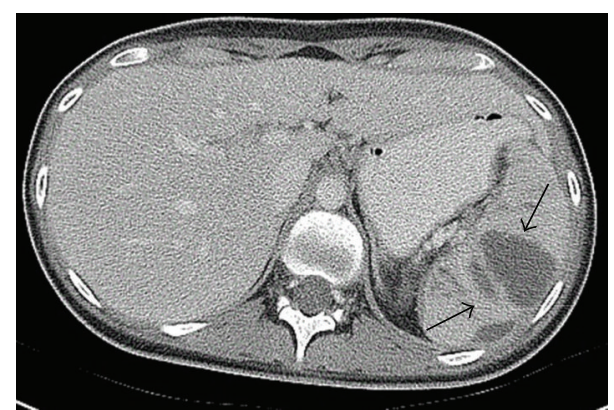

Figure 1: Splenic abscesses noted on CT scan (arrows).

abdominal CT one month later showed increased size and number of the splenic lesions and a small intra-abdominal fluid collection near the cecum. During this time, all aerobic, anaerobic, mycobacterial, and fungal blood cultures remained negative as well as a transesophageal echocardiogram for endocarditis. The CT-guided aspiration of the abdominal fluid collection was sent for cultures and cytology and returned negative for any infectious or malignant process.

Ultimately, all antibiotics were discontinued due to side effects and lack of clinical improvement. The extensive workup included serologic testing for infectious processes, skin biopsies, thorough rheumatologic tests, and comprehensive immunologic evaluation for primary immune deficiencies including chronic granulomatous diseases and secondary immune deficiencies as well as an upper endoscopy which was unrevealing. A colonoscopy with biopsy revealed regions of focal mild acute ileitis with villous blunting and fibrosis. The right colon demonstrated mildly increased lymphoplasmacytic infiltration in the lamina propria. The left colon and rectum displayed acute cryptitis. These findings indicated acute colitis and proctitis consistent with Crohn's disease. An elevated $\operatorname{IgA}$ and $\operatorname{IgG}$ anti-Saccharomyces cerevisiae antibodies (ASCA) and negative perinuclear antineutrophil cytoplasmic antibodies (p-ANCA) further supported this diagnosis. The patient was started on oral corticosteroids and demonstrated symptomatic improvement during her hospital stay with eventual resolution of splenic lesions within 6 months (Figure 2). She was followed closely by gastroenterology within the first few months of her diagnosis and was transitioned from prednisone to azathioprine with continued resolution of both skin and splenic findings with no further evolution of symptoms. A repeat colonoscopy performed 8 months after the initial diagnosis was normal. The patient continues to be followed by gastroenterology annually.

\section{Discussion}

This report describes a young female with recurrent aseptic pustular skin lesions as well as sterile splenic abscesses refractory to antibiotic treatments. The results of the small and large bowel biopsies as well as serology suggested Crohn's

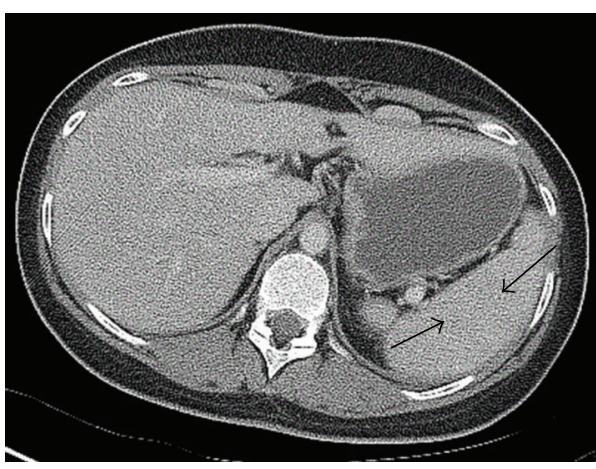

FIGURE 2: Resolution of the patient's splenic abscesses 6 months following treatment initiation (previously effected regions denoted by arrows).

disease. The responses to prednisone and azathioprine offered additional support for the diagnosis of IBD.

PubMed and OVID were searched for the current English literature using the following keywords: splenic abscess, IBD, Crohn's disease, and aseptic splenic abscess. The search revealed case reports and case series with a variable number of patients, ranging from 1 to 287 [1, 6, 13-20]. Splenic abscesses are uncommon and, based on autopsy reviews, the incidence between 0.14 and $0.7 \%$ has been reported $[1,2]$. Aseptic splenic abscesses are rare, with the majority of cases being reported by European countries [3-5].

The signs and symptoms of splenic abscess are nonspecific and may include fever, abdominal pain, and leukocytosis $[9,17,21,22]$. The causative organisms in most reports are aerobic bacteria such as Streptococcus, Staphylococcus, and Enterococcus or anaerobic bacteria such as Escherichia coli [9-12]. A group in Spain identified Mycobacterium tuberculosis (MTB) as the causative pathogen in $36.4 \%$ of 22 cases [11]. Two groups from Japan and one group from Taiwan noted that gram-negative bacillus accounted for $46.7 \%-60 \%$ of their cases $[6,23]$. Predisposing factors, such as diabetes mellitus, malignancies, immunosuppressive medications, or immunodeficiency disorders, often play a role in the development of splenic abscess and should be considered when a splenic abscess is found $[8,21,22,24-26]$.

Few case series have specifically looked at patients with sterile splenic abscesses. One case series found M-TB to cause culture negative splenic abscesses. The case series mentioned above reported that approximately $13 \%$ of patients have had sterile splenic abscesses [27]. A report from France outlined the clinical course in 30 patients. The mean age at diagnosis was 29 years and main clinical manifestations were fever, abdominal pain, and weight loss. Twenty-two of these patients had leukocytosis and twenty-one had IBD diagnosed by colonoscopy and/or barium swallow. Serological studies with elevated CRP $>6$, antinuclear antibodies, and rheumatoid factor were found in this group. Tests for ANCA and anti-Saccharomyces cerevisiae antibodies (ASCA) were performed. Of the 21 patients diagnosed with IBD, the diagnosis preceded the splenic abscesses in 7 cases, was identified at the same time as the abscesses in 7 cases, and appeared 
secondarily in 7 cases [13]. A recent report published discussed a case of a 15-year-old girl who developed multiple aseptic abscesses. She was treated with a combination of metronidazole, meropenem, and prednisone $2 \mathrm{mg} / \mathrm{kg}$ therapy with symptom resolution. The subject was subsequently found to have autoimmune thyroiditis [28].

There are scarce reports listing splenic abscesses as the first sign of Crohn's disease. A recent case report in 2010 describes a patient with aseptic splenic abscesses as the first manifestation of Crohn's disease. Unlike our case, that patient was male and in his 60s. He experienced recurring splenic abscesses unresponsive to antibiotic therapy and had a negative workup for infections, malignancies, lymphoproliferative processes, and immunodeficiencies. Crohn's disease was eventually diagnosed after an enteroscopy with biopsy demonstrated cryptitis, inflammatory cellularity in the lamina propria, and epithelioid granuloma consistent with Crohn's disease [20].

\section{Conclusion}

The presentation of pustular skin lesions, splenic abscesses, leukocytosis, elevated ESR, and CRP suggested an infectious etiology in our patient. After an extensive negative workup for infectious etiologies and a thorough immunological evaluation in addition to failure to respond to antibiotic therapy, an alternative diagnosis was suspected. The results of the colonoscopy with biopsy and serology were consistent with the diagnosis of Crohn's disease. While this condition has been documented internationally, there has been little consideration of IBD on the differential diagnosis for patients with aseptic splenic abscesses in the United States. Colonoscopies with biopsies are needed for diagnosis. The institution of immunosuppressive medications can lead to symptomatic improvement and resolution of splenic lesions that are due to IBD $[11,20,21,29]$.

\section{Conflict of Interests}

The authors of this paper declare there is no conflict of interests regarding the publication of this paper.

\section{Acknowledgments}

The authors of this paper would like to thank Dr. Mohammad Reza and Dr. Erin Banta for their contributions.

\section{References}

[1] J. D. Chulay and M. R. Lankerani, "Splenic abscess. Report of 10 cases and review of the literature," The American Journal of Medicine, vol. 61, no. 4, pp. 513-522, 1976.

[2] S. E. Reid and S. J. Lang, "Abscess of the spleen," The American Journal of Surgery, vol. 88, no. 6, pp. 912-917, 1954.

[3] S. Gupta, O. Singh, A. Hastir, S. Shukla, and R. K. Mathur, "Splenic abscesses: reports of two cases with review of the literature," Annals of Tropical Medicine and Public Health, vol. 5, no. 3, pp. 273-277, 2012.
[4] E. G. Bromley and S. D. Leonard, "Isolated aseptic liver abscesses in a patient with ulcerative colitis," Proceedings of UCLA Healthcare, vol. 17, 2013.

[5] S. Shuba, Y. C. Lakshmanakumar, and S. R. Prasad, "Splenic abscesses-a study of 4 cases," Advance Laboratory Medicine International, vol. 1, no. 3, pp. 55-60, 2011.

[6] K.-C. Chang, S.-K. Chuah, C.-S. Changchien et al., "Clinical characteristics and prognosis factors of splenic abscess: a review of 67 cases in a single medical center of Taiwan," World Journal of Gastroenterology, vol. 12, no. 3, pp. 460-464, 2006.

[7] J. Llenas-García, M. Fernández-Ruiz, L. Caurcel, A. EnguitaValls, J. Vila-Santos, and J.-M. Guerra-Vales, "Splenic abscess: a review of 22 cases in a single institution," European Journal of Internal Medicine, vol. 20, no. 5, pp. 537-539, 2009.

[8] G. Ferraioli, E. Brunetti, R. Gulizia, G. Mariani, P. Marone, and C. Filice, "Management of splenic abscess: report on 16 cases from a single center," International Journal of Infectious Diseases, vol. 13, no. 4, pp. 524-530, 2009.

[9] A. R. Alvi, S. Kulsoom, and G. Shamsi, "Splenic abscess: outcome and prognostic factors," Journal of the College of Physicians and Surgeons Pakistan, vol. 18, no. 12, pp. 740-743, 2008.

[10] N. Nelken, J. Ignatius, M. Skinner, and N. Christensen, "Changing clinical spectrum of splenic abscess. A multicenter study and review of the literature," The American Journal of Surgery, vol. 154, no. 1, pp. 27-34, 1987.

[11] S. I. Pelton, J. Y. Kim, and R. L. Kradin, "Case 27-2006: a 17-yearold boy with fever and lesions in the liver and spleen," The New England Journal of Medicine, vol. 355, no. 9, pp. 941-948, 2006.

[12] M. Yilmaz, F. Arslan, O. Baskan, and A. Mert, "Splenic abscess due to brucellosis: a case report and a review of the literature," vol. 20, pp. 68-70, 2014.

[13] M. F. Andre, J. C. Piette, J. L. Kemeny et al., "Aseptic abscesses: a study of 30 patients with or without inflammatory bowel disease and review of the literature," Medicine (Baltimore), vol. 86, no. 3, pp. 145-161, 2007.

[14] S. R. Choudhury, P. R. Debnath, P. Jain et al., "Conservative management of isolated splenic abscess in children," Journal of Pediatric Surgery, vol. 45, no. 2, pp. 372-375, 2010.

[15] C. H. Chun, M. J. Raff, L. Contreras et al., "Splenic abscess," Medicine, vol. 59, no. 1, pp. 50-65, 1980.

[16] L. L. Ooi and S. S. Leong, "Splenic abscesses from 1987-1995," The American Journal of Surgery, vol. 174, no. 1, pp. 87-93, 1997.

[17] K. N. Rattan, Y. S. Kadian, V. Saroha, and N. Jindal, "Splenic abscess in children: a report of three patients," African Journal of Paediatric Surgery, vol. 6, no. 2, pp. 106-109, 2009.

[18] A. E. Billings, "Abscess of the spleen," Annals of Surgery, vol. 88, no. 3, pp. 416-428, 1928.

[19] R. S. Choudhury, C. Rajiv, S. Pitamber, S. Akshay, and S. Dharmendra, "Management of splenic abscess in children by percutaneous drainage," Journal of Pediatric Surgery, vol. 41, no. 1, pp. E53-E56, 2006.

[20] S. Calzado, M. Navarro, I. Puig, and B. Font, "Splenic abscess as the first manifestation of Crohn's disease," Journal of Crohn's and Colitis, vol. 4, no. 6, pp. 703-704, 2010.

[21] C.-C. Tung, F.-C. Chen, and C.-J. Lo, "Splenic abscess: an easily overlooked disease?” American Surgeon, vol. 72, no. 4, pp. 322325, 2006.

[22] M. L. Sternberg and N. P. Lisenbee, "Splenic abscesses," Journal of Emergency Medicine, vol. 44, no. 1, pp. e83-e84, 2013.

[23] E. Zerem and J. Bergsland, "Ultrasound guided percutaneous treatment of splenic abscesses: the significance in treatment of 
critically ill patients," World Journal of Gastroenterology, vol. 12, no. 45, pp. 7341-7345, 2006.

[24] R. M. Hatley, J. S. Donaldson, and J. G. Raffensperger, "Splenic microabscesses in the immune-compromised patient," Journal of Pediatric Surgery, vol. 24, no. 7, pp. 697-699, 1989.

[25] M. Kang, N. Kalra, M. Gulati, A. Lal, R. Kochhar, and A. Rajwanshi, "Image guided percutaneous splenic interventions," European Journal of Radiology, vol. 64, no. 1, pp. 140-146, 2007.

[26] A. K. Singh, S. Shankar, D. A. Gervais, P. F. Hahn, and P. R. Mueller, "Image-guided percutaneous splenic interventions," Radiographics, vol. 32, no. 2, pp. 523-534, 2012.

[27] C. Birkenmaier, U. Reischl, and B. Stübinger, "Sterile abscess: a surprise diagnosis?" Scandinavian Journal of Infectious Diseases, vol. 38, no. 1, pp. 15-18, 2006.

[28] A. J. Jordan, K. P. Becker, M. Sertemir et al., "Multiple aseptic splenic abscesses in a 15 year old patient," BMC Gastroenterology, vol. 14, no. 20, pp. 1-4, 2014.

[29] D. K. Podolsky, "Inflammatory bowel disease," The New England Journal of Medicine, vol. 325, no. 13, pp. 928-937, 1991. 


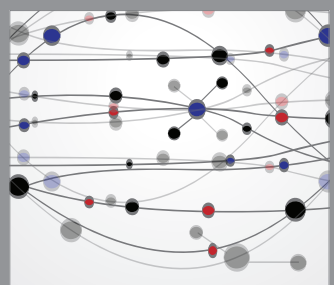

The Scientific World Journal
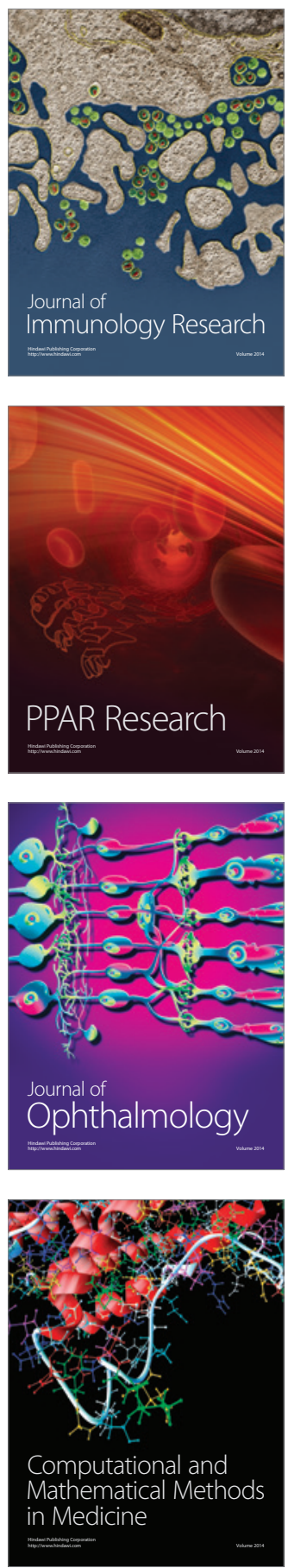

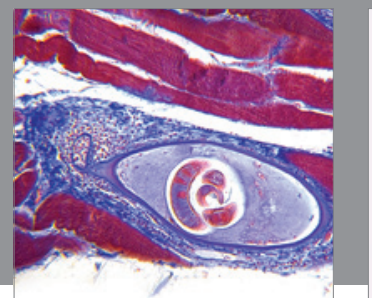

Gastroenterology

Research and Practice
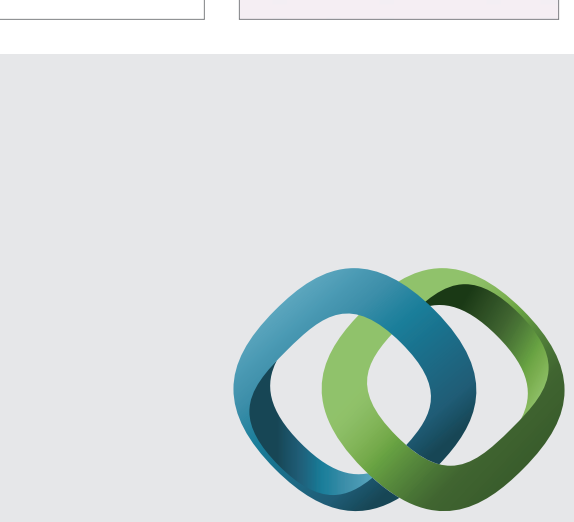

\section{Hindawi}

Submit your manuscripts at

http://www.hindawi.com
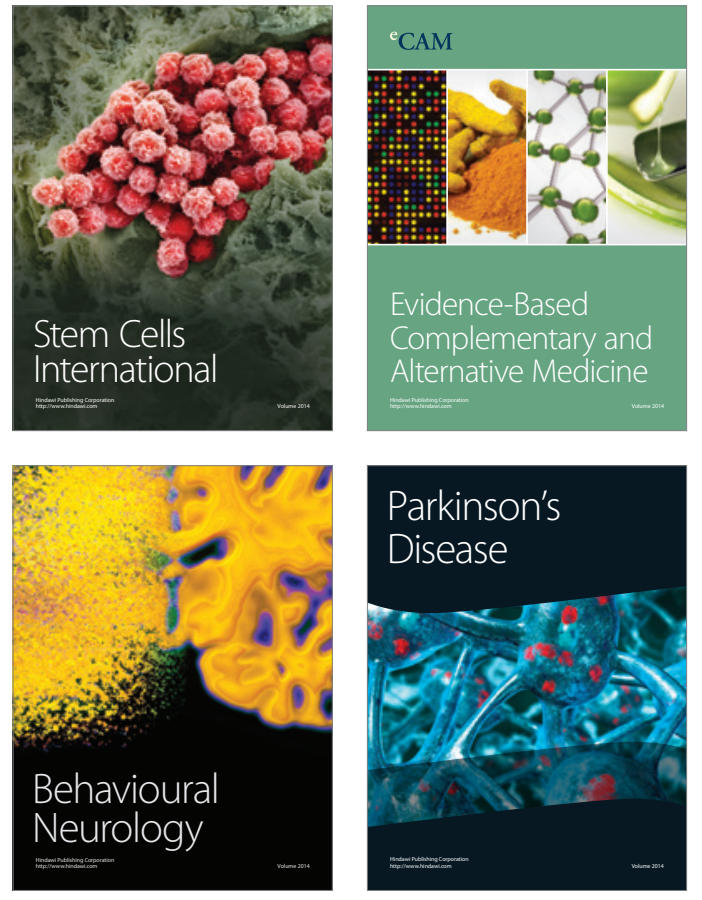
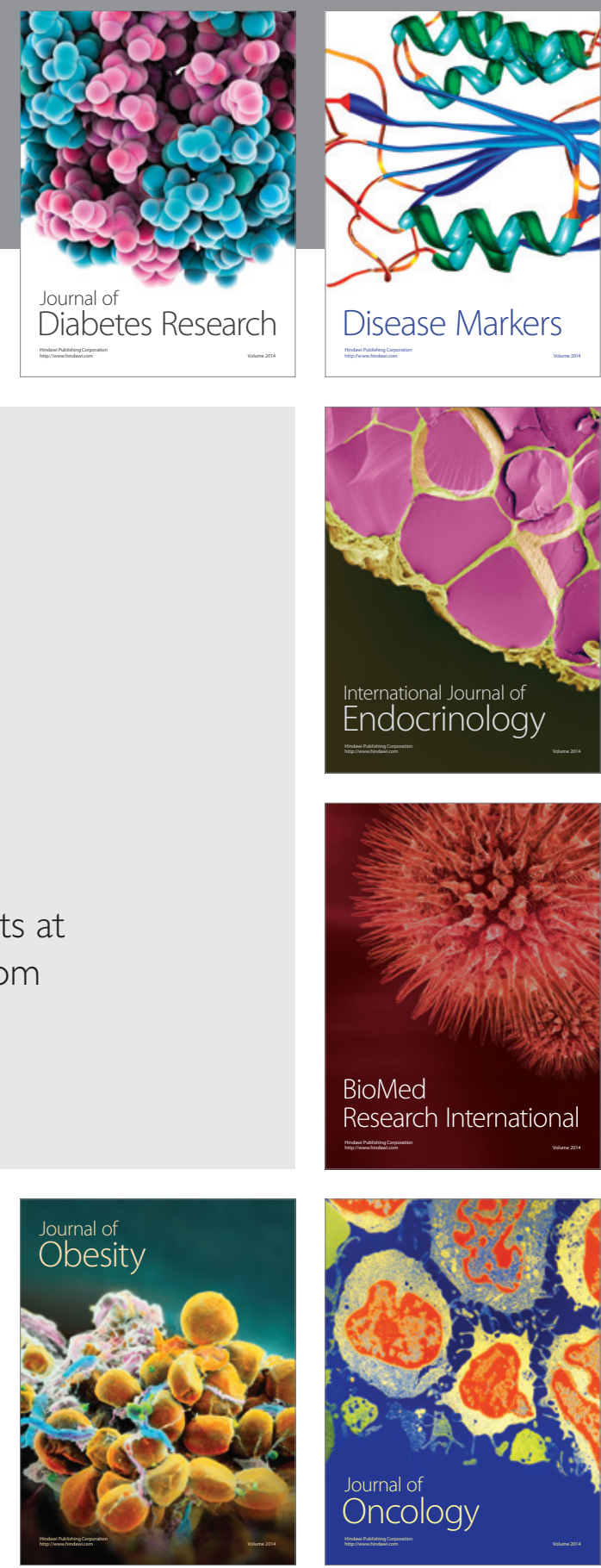

Disease Markers
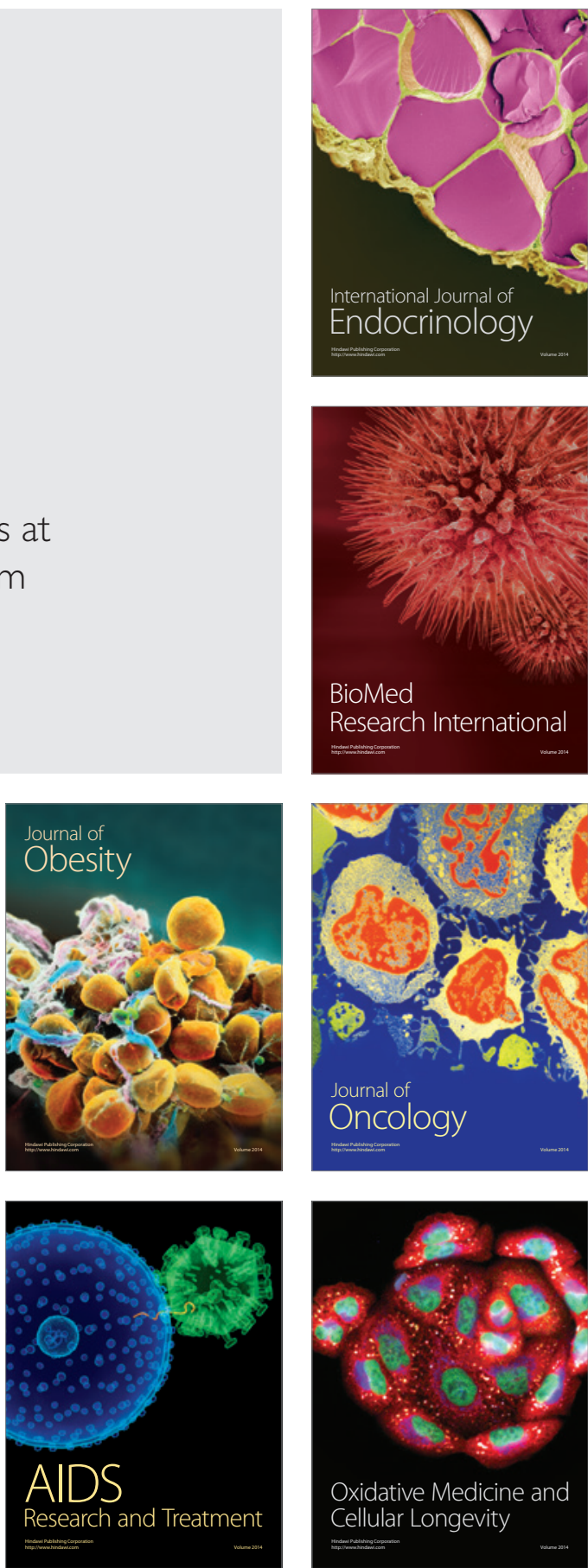